\section{THU0205 THE HEMATOPOIETIC STEM CELLS (HSCS) IN SYSTEMIC LUPUS ERYTHEMATOSUS (SLE) REPROGRAM THEIR TRANSCRIPTOME: IMPLICATIONS FOR THE PATHOGENESIS OF THE DISEASE}

Aggelos Banos $^{1}$, Maria Grigoriou ${ }^{1}$, Anastasia Filia ${ }^{1}$, Pavlos Pavlidis ${ }^{2}$, Lina Giannouli ${ }^{3}$, Vassiliki Karali ${ }^{4}$, Dionisis Nikolopoulos ${ }^{4}$, Antigoni Pieta ${ }^{4}$ loannis Mitroulis ${ }^{5}$, Panayotis Verginis ${ }^{1}$, Dimitrios Boumpas ${ }^{4} .{ }^{1}$ Biomedical Research Foundation of the Academy of Athens, Laboratory of Immune Regulation and Tolerance Autoimmunity and Inflammation, Athens, Greece; ${ }^{2}$ Foundation for Research and Technology-Hellas, Institute of Computer Science, Herakleion, Greece; ${ }^{3}$ National and Kapodistrian University of Athens, 2nd Department of Medicine, Ippokrateion University Hospital, Athens, Greece; ${ }^{4}$ National and Kapodistrian University of Athens, 4th Department of Medicine, Attikon University Hospital, Athens, Greece, ${ }^{5}$ Democritus University of Thrace, Laboratory of Molecular Hematology, Department of Medicine and First Department of Internal Medicine, University Hospital of Alexandroupolis, Alexandroupolis, Greece

Background: In SLE, all terminally differentiated blood cells demonstrate an aberrant phenotype. HSCs the most primitive cell type of the hematopoietic lineage when exposed within the bone marrow (BM) to adjuvants and inflammatory mediators change their transcriptional landscape and this may persist in the HSCs circulating in the peripheral blood or those infiltrating peripheral tissues. Within peripheral tissues these reprogramed HSCs differentiate into myeloid cells mounting enhanced protective or aberrant immune responses ${ }^{1}$.

Objectives: To dissect whether aberrant phenotypes of blood cells in SLE could be traced back to HSCs and explore how the inflammatory environment of SLE shapes the HSC differentiation process.

Methods: We analyzed the transcriptional alterations (genetic or epigenetic) of $\mathrm{CD}_{3} 4^{+}$cells in the BM of SLE patients, compared it to healthy individuals and the NZB/W lupus mice at the onset of disease (6 months). $\mathrm{CD} 4^{+}$cells were isolated from $\mathrm{BM}$ aspirates and peripheral blood of SLE patients $(n=8)$ and healthy subjects $(n=2)$ with magnetic separation (Stem Cell Technologies). mRNA was extracted and libraries were prepared. Sequencing was performed in NextSeq Illumina Platform. Alignment in human genome v.38 was done by Star package and differential expression analysis was performed by edgeR algorithm. Genes with $\mathrm{FC} \geq 1.5 / \leq-1.5, \quad F D R \leq 0.05$ were considered statistically significantly up-/ down-regulated, respectively. Heatmaps were constructed in R, GO/Pathway Analysis and enrichment analysis were performed in ClueGo, RNEA, GeneMania and GSEA, respectively.

Results: Overlaying the transcriptome of BM-derived $\mathrm{CD}_{3} 4^{+}$of SLE patients and healthy subjects, we identified in total 598 differentially expressed genes(DEGs) (82 up-/514 down-regulated in SLE). DEGs participate in hematopoietic cell lineage fate, regulation of stem cell differentiation, cell adhesion and cell cycle regulation. We also found evidence for cell cycle checkpoints signature which drives HSCs to experience replication stress and activate ATR pathway. Comparison of $\mathrm{CD}_{3} 4^{+}$profile between severe-moderate SLE reveals a prominent neutrophilic signature in severe disease. Comparative transcriptomic analysis of human vs murine SLE revealed a panel of common genes again related to cell proliferation, differentiation and platelet activation.

Conclusion: HSCs in SLE patients and murine lupus reprogram their transcriptome in response to the inflammatory milieu within the $\mathrm{BM}$, thus exiting from dormancy, differentiating to myeloid cells and mounting a DNA damage response to the replication stress. This activated phenotype renders HSCs both susceptible to cell exhaustion while at the same time priming them and their progenies towards enhanced immune responses.

\section{REFERENCES:}

[1] Mitroulis, loannis, et al. "Modulation of myelopoiesis progenitors is an integral component of trained immunity." Cell 172.1 (2018): 147-161.

[2] Kaufmann, Eva, et al. "BCG educates hematopoietic stem cells to generate protective innate immunity against tuberculosis." Cell 172.1 (2018): 176-190.

Acknowledgement: This work was supported by FOREUM, IKY and ERC-Advanced Grant.

Disclosure of Interests: None declared

DOI: 10.1136/annrheumdis-2019-eular.5421

\section{THU0206 \\ ANTI-D4GDI ANTIBODIES ACTIVATE PLATELETS IN VITRO: A POSSIBLE LINK WITH THROMBOCYTOPENIA IN PRIMARY ANTIPHOSPHOLIPID SYNDROME}

cristiana barbati, Lucia Stefanini, Tania Colasanti, Enrica Cipriano, Alessandra Ida Celia, Gianna Gabriele, Marta Vomero, Fulvia Ceccarelli, Francesca Spinelli, Annacarla Finucci, Annacarla FinucciGiovanni Orso, Carlo Perricone, Mariangela Speziali, Fabrizio Conti, Francesco Violi, Guido Valesini,

Cristiano Alessandri. sapienza università di roma, dipartimento di medicina interna e specialità mediche, Rome, Italy

Background: Thrombocytopenia is a manifestation associated with Primary Antiphospholipid Syndrome (PAPS) and many studies have stressed the leading role played by platelets in the pathogenesis of Antiphospholipid Syndrome (APS) (1). Platelets are highly specialized cells and their activation involves a series of rapid rearrangements of the actin cytoskeleton (2). Recently, we described the presence of autoantibodies against D4GDI (Rho GDP Dissociation Inhibitor Beta, ARHGDIB) in the serum of a large subset of SLE patients and we observed that anti-D4GDI antibodies activated the cytoskeleton remodeling of lymphocytes by inhibiting D4GDI and allowing the upregulation of Rho GTPases, such as Rac1(3). Proteomic and transcriptomic studies indicate that D4GDI is very abundant in platelets and small GTPases of the $\mathrm{RHO}$ family are critical regulators of actin dynamics in platelets (4).

Objectives: The aim of the present study was to evaluate the presence of anti-D4GDI antibodies in PAPS sera and whether they can affect platelet activation, contributing to the thrombotic events and the thrombocytopenia of PAPS patients.

Methods: 38 PAPS patients diagnosed according to the 2006 Sydney classification criteria were enrolled from the Lupus Clinic of the Sapienza University of Rome. 20 normal healthy subjects (NHS) served as controls. Sera were stored at $-20{ }^{\circ} \mathrm{C}$ to performed an ELISA test using commercial D4GDI protein. Anti-D4GDI antibodies were purified from PAPS sera and used for in vitro treatment of platelets purified from NHS. Flow cytometry analysis was performed to determination of integrin $\alpha \mathrm{llb} \beta 3$ activation, a well-established marker of platelet activation and adhesion.

Results: We identified anti-D4GDI antibodies in sera from 18/38 (47\%) patients with PAPS, but in no sera from NHS. Dividing the patients with PAPS according to the presence or absence of thrombocytopenia, we found a significant association between this hematologic manifestation and a higher titer of anti-D4GDI antibodies. Our in vitro results show a significant $30 \%$ increase in the activation of integrin $\alpha$ llb 33 upon stimulation of platelets from healthy donors preincubated with the antibody anti-D4GDI purified from the serum of APS patients. Interestingly the antibody does not only increase the overall integrin activation but also the rate/speed of integrin activation.

Conclusion: We demonstrated that antibodies anti-D4GDI are present in the sera of PAPS patients and can prime platelet activation. Thus explaining, at least in part, the pro-thrombotic state and the thrombocytopenia of PAPS patients. These findings may lead to improved diagnosis and treatment of APS.

\section{REFERENCES}

[1] Shechter Y, Tal Y, Greenberg A, Brenner B. Platelet activation in patients with antiphospholipid syndrome. Blood Coagul Fibrinolysis. 1998 Oct;9 (7):653-7.

[2] Seong-Hoon Yun, Eun-Hye Sim, Ri-Young Goh, Joo-In Park, and Jin Yeong Han. Platelet Activation: The Mechanisms and Potential Biomarkers. BioMed Research International,Volume 2016, Article ID 9060143. Review

[3] Barbati C, Alessandri C, Vomero M, Vona R, Colasanti T, Vacirca D, Camerini S, Crescenzi M, Pendolino M, Truglia S, Conti F, Garofalo T, Sorice M, Pierdominici M, Valesini G, Malorni W, Ortona E.Autoantibodies specific to D4GDI modulate Rho GTPase mediated cytoskeleton remodeling and induce autophagy in T lymphocytes. J Autoimmun. 2015 Apr:58:78-89.

[4] Rowley JW, et al. Genome-wide RNA-seq analysis of human and mouse platelet transcriptomes. Blood. 2011;118(14).

Disclosure of Interests: None declared

DOI: 10.1136/annrheumdis-2019-eular.4058 